\title{
PERCEPCIÓN DEL CAMBIO CLIMÁTICO DE LOS AGRICULTORES ANDALUCES M.M. Delgado-Serrano ${ }^{\mathrm{a}}$, M. Rodríguez-Entrena ${ }^{\mathrm{a}}$-y M. Castillo ${ }^{\mathrm{a} *}$
}

\author{
${ }^{a}$ WEARE-Water, Environmental, and Agricultural Resources Economics Research Group, Departamento \\ de Economía Agraria, Finanzas y Contabilidad. Universidad de Córdoba (mmdelgado@uco.es; \\ mrentrena@uco.es; eslcaqum@uco.es )
}

\section{Resumen}

La agricultura andaluza, por su situación geográfica, es especialmente vulnerable al cambio climático (CC). La literatura científica muestra que los agricultores más conscientes del $\mathrm{CC}$ suelen ser más proclives a adaptarse. Sin embargo, actualmente no existe ningún análisis específico sobre la percepción del CC de los agricultores andaluces. Este trabajo pretende suplir esta carencia, con el objetivo de identificar los factores que modelan la disposición a decidir y actuar frente al CC. Para ello se realiza una encuesta a agricultores de diferentes sistemas agrarios existentes en la región. Del análisis estadístico exploratorio realizado en esta fase preliminar se constata la existencia de relaciones de relevancia predictiva entre la disponibilidad a la adaptación al CC y la creencia en la ocurrencia del fenómeno, los valores relacionados con la sostenibilidad y el CC, así como la percepción de los riesgos asociados. Por otra parte se constata la importancia de la formación, el relevo generacional y la inversión en tecnología.

Palabras clave: cambio climático; percepción; agricultores; Andalucía

\section{Introducción y objetivos}

La agricultura andaluza, por su situación geográfica, es especialmente vulnerable al cambio climático (CC). Siendo el sector agrario especialmente estratégico en Andalucía, es de vital importancia que éste sea capaz de acometer las acciones tanto de adaptación como de mitigación que el reto climático requiere. La literatura científica en este campo muestra que los agricultores más conscientes de la ocurrencia del CC suelen ser más proclives a realizar cambios en sus sistemas de explotación para adaptarse a las nuevas condiciones climáticas. Sin embargo, actualmente, no existe ningún análisis específico sobre la percepción del CC de los agricultores andaluces.

Este trabajo pretende suplir esta carencia, contribuyendo así al análisis de la resiliencia y vulnerabilidad del sector agrario andaluz frente al desafío del CC. El objetivo de este trabajo es, en primer lugar, determinar el grado de percepción del CC así como la disponibilidad a adoptar estrategias de adaptación por parte de los agricultores andaluces. En una segunda fase, se pretende determinar los factores que afectan a la percepción de los riesgos del CC.

\section{Metodología}

Para la consecución de los objetivos planteados se desarrolló una encuesta en línea utilizando la plataforma online de Microsoft Forms. La encuesta fue difundida a través de cooperativas, asociaciones de productores, instituciones y empresas ligadas al sector productor, intentando abarcar los distintos sistemas agrarios representativos de la agricultura andaluza. Los agricultores cumplimentaron los cuestionarios de manera autónoma vía ordenador o móvil. Para el diseño del cuestionario y la selección de las preguntas se realizó una revisión de la literatura centrada en la percepción del CC por parte de los agricultores (Woods et al., 2017; Hyland et al., 2016; Li et al., 2017; Arbuckle et al., 2015). En él se recogen aspectos socioeconómicos del agricultor y de la explotación, así como 54 ítems que definen las variables latentes analizadas en este estudio: experiencia de cambios en el clima; creencia en el CC; valores éticos y sociales; confianza en las fuentes de información; percepción de riesgos; barreras percibidas para la adaptación al CC; disponibilidad de adaptación; y probabilidad de adoptar cambios en la explotación (cuadro 1). Un total de 278 cuestionarios han sido respondidos hasta la fecha. En este trabajo se presenta un primer análisis estadístico exploratorio de los datos obtenidos en las encuestas.

\section{Resultados}

El cuadro 1 presenta el resumen de los ítems encuestados para cada variable latente considerada. La mayor parte de los agricultores ha experimentado en los últimos años cambios en la duración de las estaciones, subida de temperaturas, menores lluvias y escasez de agua. Un 15,5\% de los agricultores considera que algunos de los cambios climáticos beneficia a los cultivos, como son menores heladas, menores lluvias en 
la recolección, o ciclos de producción más cortos. El apoyo a la afirmación “el cambio climático está ocurriendo" es bastante amplio, recibiendo una puntuación media de 5,83 (escala Likert 1-7). También existe bastante apoyo a la afirmación de que los ciudadanos en general y el modelo de consumo es el responsable del CC, aunque la actividad agraria es escasamente responsable. Garantizar la viabilidad económica es la afirmación con mayor grado de acuerdo. En cuanto a las fuentes de información sobre CC que los agricultores consideran fiables, destaca la comunidad científica, con un fuerte consenso (el 92,1\% confía en esta fuente). En el extremo opuesto están el Gobierno (solo el 18,3\%), los medios de comunicación $(22,7 \%)$ e internet y redes sociales $(30,2 \%)$.

El CC se percibe como un riesgo, con un valor medio superior a 4 en casi todos los ítems considerados, que recogen posibilidades de pérdidas de rentabilidad y comparativas con otros tipos de riesgos de mercado y financieros. También se perciben claramente barreras a la adaptación, destacando especialmente la relativa a la incertidumbre sobre las ayudas PAC y los precios, pero también, sobre los efectos reales del CC y sobre las tecnologías disponibles. No obstante, los agricultores muestran una considerable disponibilidad a la adaptación, aunque ello represente mayores costes (puntuación media de 4,73). Esta disponibilidad se concreta en unas probabilidades declaradas de afrontar cambios tecnológicos, que son relativamente altas para prácticas de conservación del suelo, ahorro de agua y energías renovables.

El cuadro 2 presenta las correlaciones entre las variables que han resultado estadísticamente significativas. Todas las variables latentes están correlacionadas, aunque la percepción de barreras a la adaptación solo se correlaciona con los valores y la percepción de riesgos. Algunas de las relaciones encontradas entre las características socio-económicas del agricultor y de la explotación con las variables analizadas son:

- Mayor confianza en fuentes de información por parte de las agricultoras (el 19,1\% de la encuesta).

- El nivel educativo y la formación agraria se relacionan con un menor nivel de riesgo percibido y, el primero, con menor percepción de barreras y menor confianza en la fuentes de información.

- La dependencia de los ingresos agrarios se relaciona positivamente con la creencia en el CC y la disponibilidad de adaptación.

- La probabilidad de relevo generacional se relaciona positivamente con la creencia, valores, confianza, disponibilidad y probabilidad de cambio.

- Por orientación productiva, los arroceros, cultivadores de frutas y hortalizas y de viñedo, muestran mayor confianza. La menor confianza se da entre los ganaderos.

- La percepción del riesgo es mayor en los cultivadores de arroz y de viñedo y menor en frutas y hortalizas.

\section{Conclusiones}

La percepción de la ocurrencia cierta del CC resulta evidente entre los agricultores andaluces. Como principales predictores de la disposición a adaptarse a los fenómenos aparejados al CC destacan la creencia en su ocurrencia, los valores del agricultor relacionados con la sostenibilidad y el CC así como la percepción de los riesgos asociados. Estos mismos tres factores son los que muestran un mayor poder predictivo en la probabilidad de adoptar tecnologías para hacer frente al CC incluso incurriendo en mayores costes de explotación. Respecto a este último aspecto también destacan como predictores el haber experimentado en los últimos años fenómenos adversos relacionados con el CC, la probabilidad de relevo generacional, el porcentaje de ingresos agrarios y el nivel de especialización tecnológica. Estos resultados pueden ayudar a la formulación de políticas que contribuyan a una mayor adaptación y resiliencia de la agricultura andaluza al CC. En este sentido, una mayor difusión de la realidad del CC entre los agricultores puede ayudar a mejorar la creencia y, por tanto, la probabilidad de adaptación al mismo. De igual modo, unas ayudas PAC más dirigidas a agricultores profesionales (que dependen de los ingresos agrarios, con probabilidad de relevo generacional y adecuado nivel tecnológico) podría mejorar la capacidad de adaptación al CC.

\section{Bibliografía}

Arbuckle Jr, J. G., Morton, L. W., \& Hobbs, J. (2015). Understanding farmer perspectives on climate change adaptation and mitigation: The roles of trust in sources of climate information, climate change beliefs, and perceived risk. Environment and behavior, 47(2): 205-234.

Hyland, J. J., Jones, D. L., Parkhill, K. A., Barnes, A. P., \& Williams, A. P. (2016). Farmers' perceptions of climate change: identifying types. Agriculture and Human Values, 33(2): 323-339.

Li, S., Juhász-Horváth, L., Harrison, P. A., Pintér, L., \& Rounsevell, M. D. (2017). Relating farmer's perceptions of climate change risk to adaptation behaviour in Hungary. Journal of environmental management, 185: 21-30.

Woods, B. A., Nielsen, H. Ø., Pedersen, A. B., \& Kristofersson, D. (2017). Farmers' perceptions of climate change and their likely responses in Danish agriculture. Land use policy, 65: 109-1 
Cuadro 1. Resumen de valores en ítems de las variables encuestadas

\begin{tabular}{|c|c|c|}
\hline Variables & Tipo & Valores \\
\hline Experiencia: En su experiencia como agricultor ha observado en los últimos 10 años: & Nominal & $0=$ No; 1 = Sí \\
\hline mayor frecuencia de lluvias torrenciales e inundaciones & & Sí: $57,9 \%$ \\
\hline mayor frecuencia de granizadas & & $28,4 \%$ \\
\hline que llueve menos & & $86,3 \%$ \\
\hline cambios en la duración de las estaciones (verano o invierno) & & $92,4 \%$ \\
\hline mayor temperatura media & & $89,6 \%$ \\
\hline mayor frecuencia de sequías & & $80,6 \%$ \\
\hline mayor frecuencia de plagas y enfermedades & & $56,8 \%$ \\
\hline acortamiento de los ciclos de cultivo & & $67,6 \%$ \\
\hline mayor escasez de agua & & $80,6 \%$ \\
\hline ¿Alguno de los fenómenos anteriores ha afectado positivamente a su explotación? & Nominal & Sí: $15,5 \%$ \\
\hline Creencias en el Cambio climático & Ordinal & Likert: $1-7$ \\
\hline El cambio climático está ocurriendo & & Media: 5,83; Desv.: 1,45 \\
\hline El modelo de consumo es el responsable de la ocurrencia del cambio climático & & 4,91 \\
\hline La agricultura y la ganadería están contribuyendo a la ocurrencia del cambio climático & & 3,14 \\
\hline $\begin{array}{l}\text { Las leyes ambientales de la UE para luchar contra el cambio climático son positivas para el futuro de m } \\
\text { explotación }\end{array}$ & & 3,68 \\
\hline
\end{tabular}

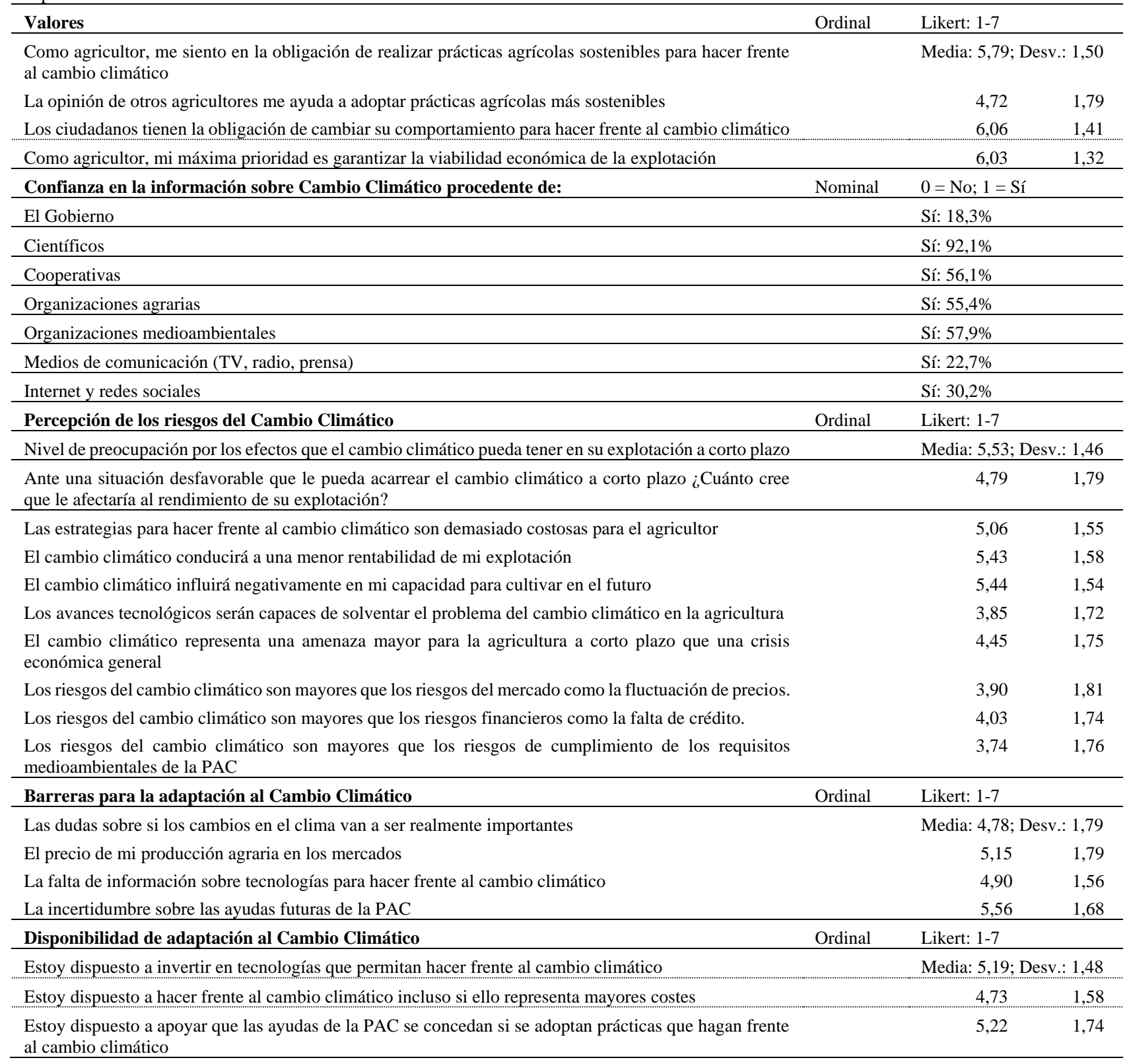




\begin{tabular}{|c|c|c|}
\hline Probabilidad de que a corto plazo realice los siguientes cambios aunque impliquen mayores costes & Ordinal & Likert: $1-7$ \\
\hline Invertir en energías renovables aplicables en la explotación & & Media: 5,56: Desv.: 1,44 \\
\hline Cambiar el manejo del suelo hacia prácticas de conservación & & 5,82 \\
\hline Cambiar el manejo del abonado para que sea más sostenible & & 5,76 \\
\hline Diversificar cultivos & & 4,85 \\
\hline Cambiar de cultivos o ganado & & 3,72 \\
\hline Cambiar el manejo de los fitosanitarios para que sea más sostenible & & 5,79 \\
\hline Invertir en sistemas de riego que permitan reducir el consumo de agua & & 5,83 \\
\hline Cambiar a sistemas de producción ecológica o integrada & & 5,23 \\
\hline Adoptar medidas agro-ambientales de la PAC & & 5,65 \\
\hline Suscribir un seguro agrario & & 4,53 \\
\hline
\end{tabular}

Fuente: Elaboración propia

Cuadro 2. Correlaciones y diferencias estadísticamente significativas ${ }^{(1)}$

\begin{tabular}{|c|c|c|c|c|c|c|c|c|c|}
\hline & & $\begin{array}{l}\text { Experien } \\
\text { cia }\end{array}$ & $\begin{array}{l}\text { Creencias } \\
\text { en CC }\end{array}$ & Valores & $\begin{array}{l}\text { Confianza } \\
\text { información }\end{array}$ & $\begin{array}{l}\text { Percepción } \\
\text { de riesgos }\end{array}$ & $\begin{array}{l}\text { Barreras } \\
\text { adaptación }\end{array}$ & $\begin{array}{l}\text { Disponib. } \\
\text { adaptación }\end{array}$ & $\begin{array}{l}\text { Probabilidac } \\
\text { de cambios }\end{array}$ \\
\hline \multirow{8}{*}{ 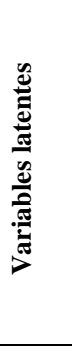 } & Experiencia & &, $213 * *$ & $270 * * *$ &, $254 * *$ & $361 * * *$ & & $201 * * *$ &, $275 * * *$ \\
\hline & Creencias en CC & & & $421 * * *$ & .256 **** & $375^{* * *}$ & & $353 * * *$ & $346 * * *$ \\
\hline & Valores & & & & $299 * * *$ & $279 * * *$ &, $131 *$ & $400 * * *$ &, $401 * * *$ \\
\hline & Confianza inf. & & & & & $.262 * * *$ & & $.180^{* * * *}$ &, $188 * * *$ \\
\hline & Percep. de riesgos & & & & & & $211 * * *$ & $356 * * *$ &, $288 * * *$ \\
\hline & Barreras adaptación & & & & & & &, $120 * *$ & \\
\hline & Disponi. adaptación & & & & & & & &, $577 * * *$ \\
\hline & Prob. de cambios & & & & & & & & \\
\hline \multirow{5}{*}{ 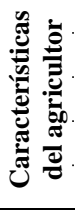 } & Sexo: Varón & & & & $-2,160 *$ & & & & \\
\hline & Formación & & & &,$- 140 *$ &,$- 197 * * *$ &,$- 122 *$ & & \\
\hline & Formación agraria & & & & & $-0,173^{* *}$ & & & \\
\hline & \% Ingresos agrarios & &, $175^{* *}$ & & & & &, $147 *$ & \\
\hline & Prob. de relevo & &, $119 *$ &, $170^{*}$ &, $144 *$ & & &, $153 *$ &, $177 * *$ \\
\hline \multirow{6}{*}{ 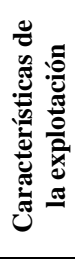 } & SAU & &,$- 183 * *$ & &,$- 170 * *$ & & & & \\
\hline & Orient. Productiva & & & & $3,376^{* * *}$ & $2,925 * *$ & & & \\
\hline & Ingresos agrarios & $-0,129 *$ & $-0,130 *$ & & & & & & \\
\hline & Ecológico/integrada & & & & & & & & $2,439^{*}$ \\
\hline & Nivel tecnológico & & & & & &, $133^{*}$ &, $121^{*}$ & \\
\hline & Asalariados fijos & & & & & & $2,271^{*}$ & & \\
\hline \multirow{3}{*}{ 莺 } & Miembro sindicato & $-2,134^{*}$ & $-2,017 *$ & & & & & & \\
\hline & Contrata seguro & & & & & & & & $2,484 *$ \\
\hline & Con préstamos & & $-2,502 *$ & & & & & & \\
\hline
\end{tabular}

(1) Valores de los estadísticos $\rho$, $t y F$, según proceda en función del análisis estadístico bivariante empleado. Se muestran los valores significativos al 0,001 (***), 0,01(**), y 0,05 (*)

Fuente: Elaboración propia. 